\title{
Detailed assignment of normal and resonant Auger spectra of Xe near the $L$ edges
}

\author{
R. Püttner, ${ }^{1, *}$ K. Jänkälä, ${ }^{2}$ R. K. Kushawaha, ${ }^{3}$ T. Marchenko, ${ }^{3,4}$ G. Goldsztejn, ${ }^{5}$ O. Travnikova, ${ }^{3,4}$ R. Guillemin, ${ }^{3,4}$ \\ L. Journel, ${ }^{3,4}$ I. Ismail, ${ }^{3}$ B. Cunha de Miranda, ${ }^{3}$ A. F. Lago, ${ }^{6}$ D. Céolin, ${ }^{4}$ M. N. Piancastelli, ${ }^{3,7}$ and M. Simon ${ }^{3,4}$ \\ ${ }^{1}$ Fachbereich Physik, Freie Universität Berlin, Arnimallee 14, D-14195 Berlin, Germany \\ ${ }^{2}$ Nano and Molecular Systems Research Unit, University of Oulu, P.O. Box 3000, 90014 Oulu, Finland \\ ${ }^{3}$ Sorbonne Universités, UPMC Univ Paris 6, CNRS, UMR 7614, Laboratoire de Chimie Physique-Matière et Rayonnement, \\ F-75005 Paris, France \\ ${ }^{4}$ Synchrotron SOLEIL, l'Orme des Merisiers, Saint-Aubin, BP 48, F-91192 Gif-sur-Yvette Cedex, France \\ ${ }^{5}$ Max-Born-Institut, Max-Born-Straße 2A, 12489 Berlin, Germany \\ ${ }^{6}$ Centro de Ciências Naturais e Humanas, Universidade Federal do ABC (UFABC), Avenida dos Estados, 5001, 09210-580, \\ Santo André, São Paulo, Brazil \\ ${ }^{7}$ Department of Physics and Astronomy, Uppsala University, Box 516, SE-75120 Uppsala, Sweden
}

(Received 24 May 2017; published 1 August 2017)

\begin{abstract}
We present a comprehensive experimental and theoretical investigation on the $L M M, L M N$, and $L N N$ normal Auger spectra of xenon, which reveal excellent agreement with theory when core-hole lifetimes of the two-hole final states are taken into account. Generally, the spectra turned out to be highly complex due to a strong overlap of the Auger transitions subsequent to $2 s_{1 / 2}^{-1}, 2 p_{1 / 2}^{-1}$, and $2 p_{3 / 2}^{-1}$ ionization. This overlap is due to the splitting of the three initial $L$ core holes and the different final $M$ and $N$ core holes being on the same order of magnitude of several hundred $\mathrm{eV}$. The Auger transitions are assigned in detail based on the theoretical results. Most of the $M M$, $M N$, and $N N$ final states are described well based on $j j$ coupling. In addition, we present a detailed assignment of the resonant $L M_{45} M_{45}$ Auger transition subsequent to the $2 s \rightarrow 6 p, 7 p$ and $2 p \rightarrow 5 d, 6 d$ excitations.
\end{abstract}

DOI: 10.1103/PhysRevA.96.022501

\section{INTRODUCTION}

After a photoexcitation or photoionization process leading to the creation of a deep core hole in atoms and molecules, one possible decay pattern is that the electron vacancy is filled by electrons from higher shells in a process which can consist of several steps according to the depth of the primary hole. Simultaneously, electrons from higher shells are emitted, known as Auger electrons.

In general, two regimes can be distinguished while investigating the decay of core-excited states across a resonance: the below- and above-threshold regions. The below-threshold region is usually characterized by relatively narrow resonant spectral features as the decay channel is indistinguishable from the direct photoionization leading to the same final states of the process. The above-threshold region includes diagram and satellite Auger decay lines. The diagram lines are the result of a core-ionization process with only one electron involved and a subsequent Auger decay process with only two electrons involved. Contrary to this, satellite Auger lines are observed when in the ionization and/or the decay process additional electrons are involved.

The study of deep-edge photoexcitation, photoionization, and decay processes in isolated atoms and molecules has recently been given a strong impulse thanks to the availability of a state-of-the-art facility at synchrotron SOLEIL, Saint Aubin, France: The GALAXIES beamline, equipped with an end station based on a dispersive-type electron-energy analyzer $[1,2]$, makes it possible nowadays to acquire photoemission and Auger spectra with optimal total experimental resolution, in the so-called "tender" $\mathrm{x}$-ray range $(2-14 \mathrm{keV})$.

*puettner@physik.fu-berlin.de
In particular, experiments can also be performed under the so-called Auger resonant Raman conditions, meaning that resonant Auger decay spectra can be measured with a total experimental resolution better than the lifetime broadening of the core-excited states [3]. Using the GALAXIES beamline this is feasible for the first time even at edges as deep as $K$ edges of second-row elements $(\mathrm{S}, \mathrm{Cl}, \mathrm{Ar})$ or $L$ edges of third-row elements (I, Xe).

We have already shed light on a wealth of phenomena which can be evidenced in this new field. In particular, in this energy range where the emitted photoelectrons can have very high kinetic energy, we have shown that recoil phenomena due to the momentum of the photoelectron become important [4]. Furthermore, super-shake-up structures related to final states with two vacancies in the core shell(s) and one excited electron can be measured [5-7]. In addition, ultrafast nuclear motion on the femtosecond and even subfemtosecond time scale [8] as well as ultrafast dissociation following core excitation and subsequent Auger cascade $[9,10]$ can be observed. Because of the very short lifetimes of the deep core-hole states such as Ar $1 s^{-1}$ and high kinetic energies of Auger electrons, large postcollision interaction (PCI) effects are also reported [11]. Finally, alternative effects such as electronic state-lifetime interference can be found [12-15].

Deep core holes have a short lifetime, and therefore large natural linewidth. If the spacing between core-excited states is of the same order of magnitude as their linewidth, interference between decay channels involving partially overlapping excited states and leading to the same final states is possible. The resulting electronic state-lifetime interference can be observed in the energy position and profile of the peaks corresponding to the different final states.

In a previous paper [13], we investigated resonant $L M_{4,5} M_{4,5}$ Auger decay of xenon following photoexcitation 
of $L_{1}, L_{2}$, and $L_{3}$ edges under resonant Raman conditions, with much improved resolution compared to previous data sets. From these measurements we extracted partial cross sections for the population of the individual final states of the resonant Auger decay. We showed the signature of electronic-state-lifetime interference effects between several coherently excited intermediate states, due to large lifetime broadening. Such interference manifests itself by final-state line narrowing or broadening, according to its constructivedestructive character, and by shifts of the maxima of the partial cross sections with respect to resonance positions. Electron recapture processes were also observed above all three photoionization thresholds. However, in the previous paper we published a simplified spectral assignment, sufficient to show general trends and to clarify the above-mentioned effects, but without fully attributing the configurations of the various final states in detail. Moreover, in a very recent publication some of the present authors reported the entire LMM normal Auger spectrum measured with photon energies below and above the $\mathrm{Xe} 1 s^{-1}$ ionization threshold at $34565 \mathrm{eV}$ in order to study the dominant role of the $K L$ emission subsequent to the creation of a hole in a deep $K$ shell [16]. In that publication the spectral features were related to the different $L$ core holes, but no detailed assignment is given.

In this work we present detailed experimental and theoretical results for the $L M M, L M N$, and $L N N$ normal Auger spectra. These spectra turned out to be rather complex, since the splitting of the three different $L$ holes of the initial state is in the same order of magnitude as the splitting of the $M$ and $N$ holes of the final state. As a consequence, in several cases the $L_{1}, L_{2}$, and $L_{3}$ Auger spectra, like $L_{3} M_{4,5} N_{4,5}$ and $L_{1} M_{4,5} M_{4,5}$, overlap partially with very similar intensities and linewidths. We, nevertheless, show a rather complete spectral assignment of the $L M M, L M N$, and $L N N$ normal Auger spectra, which we have achieved for the majority of final states. In addition, we assign in detail the spectral features of the resonant $L M_{4,5} M_{4,5}$ Auger decay subsequent to the $2 s \rightarrow 6 p, 7 p$ and $2 p \rightarrow 5 d, 6 d$ excitations. The assignments are performed on the ground of relativistic configuration interaction Dirac-Fock theoretical calculations, which show an excellent agreement with the experimental data.

\section{EXPERIMENT}

The GALAXIES beamline at SOLEIL, Saint Aubin, France, provides a monochromatic and highly focused beam with the highest possible flux in the spectral range $2.3-13 \mathrm{keV}$ and a photon-energy bandwidth between $200 \mathrm{meV}$ and $1 \mathrm{eV}$ [1]. Resonant Auger measurements near the $L$ edges of xenon were performed using the hard $\mathrm{x}$-ray photoelectron spectroscopy (HAXPES) end station [2], which allows us to study resonant Auger processes even at deep shells under resonant Raman conditions.

With the HAXPES setup, the electron kinetic-energy measurements are carried out by the newly designed EW4000 VGScienta hemispherical photoelectron analyzer. The instrument is optimized for the detection of electron kinetic energies up to $12 \mathrm{keV}$ with an expected resolution of $35 \mathrm{meV}$ for electrons having a kinetic energy of $10 \mathrm{keV}$, while providing high transmission thanks to the $60^{\circ}$ total horizontal opening angle of the lens. The electron analyzer is mounted on a $\mu$-metal-shielded $\mathrm{U} H V$ experimental chamber, and the lens system is placed perpendicularly to the beam and parallel to the electric field vector of the linearly polarized synchrotron light. The gas cell is mounted on a fully motorized four-axis manipulator located at the bottom of the main chamber.

A system of differential pumping on both entrance and exit paths of the HAXPES station ensures high-vacuum conditions in the rest of the beamline even when gas is introduced into the main chamber. During the measurements on xenon, the pressure in the chamber was maintained at $5 \times 10^{-6}$ mbar. The pressure in the gas cell cannot be measured directly; it is, however, expected to be two to three orders of magnitude higher than the background pressure. The analyzer parameters such as pass energy $(500 \mathrm{eV})$ and slit width $(400 \mu \mathrm{m})$ were fixed during the experiment, yielding a resolution of $0.5 \mathrm{eV}$. The photon bandwidth was approximately $0.6 \mathrm{eV}$ at $5 \mathrm{keV}$ photon energy, resulting in a total experimental width of approximately $0.8 \mathrm{eV}$ for the $\mathrm{Xe} 4 d^{-1}$ and $5 s^{-1}$ lines. The spectra were recorded at $0^{\circ}$ with respect to the linear polarization axis.

For the normal Auger measurements, a large window of 2500-4700 eV was used. To distinguish the Auger transitions from the different core holes unambiguously, the spectra were measured at three different photon energies, namely, 4799.2(0.7), 5118.6(0.7), and 5465.3(0.7) eV. These values are approximately $12 \mathrm{eV}$ above the ionization potentials of the $L_{3}, L_{2}$, and $L_{1}$ ionization thresholds and guarantee that in the Auger spectra only shakes during the Auger process can be observed while Auger decays of shake-up or shakeoff states during the photoionization process are absent. As recently shown for $\mathrm{H}_{2} \mathrm{~S}$, shake processes occurring during the ionization and the decay process are present well above threshold, rendering the assignment more complicated [17]. The resonant Auger spectra were recorded at different photon energies directly below the $L_{1}, L_{2}$, and $L_{3}$ threshold by using kinetic-energy windows of $45-65 \mathrm{eV}$ around the most intense $2 s^{-1} \rightarrow 3 d^{-2} 6 p$ and $2 p^{-1} \rightarrow 3 d^{-2} 5 d$ transitions.

The kinetic-energy scale was calibrated by using the known energy positions of the Ar LMM [18] and Ar KMM normal Auger spectra; the latter ones are derived from the Ar $1 s^{-1}$ ionization energy [19] and the binding energies of the $3 p^{-2}$ final states [20]. In order to calibrate the photon-energy scale, Ar $2 p^{-1}$ photoelectron spectra were obtained in the photonenergy ranges used for the actual measurements, and calibrated by using the known value of $250.79 \mathrm{eV}$ for the $\mathrm{Ar} 2 p_{1 / 2}^{-1}$ binding energy [18]. Based on the uncertainties of the kinetic energies of the Auger lines as well as the calibration procedure we estimate the uncertainty of the kinetic-energy axis of the Auger spectra on the order of $0.7 \mathrm{eV}$.

\section{THEORY}

Theoretical calculations were carried out using the relativistic configuration interaction Dirac-Fock framework, where the total atomic state functions (ASFs) are constructed as linear combinations of the same total angular momentum $J$, its projection $M$, and parity $P$. The basis of the ASFs are inherently $j j$-coupled configuration state functions (CSFs), 
formed as determinantal products of one-electron relativistic spin orbitals. The coefficients of the linear combination were solved by diagonalizing the Dirac-Coulomb-Breit Hamiltonian in the CSF basis with fixed radial one-electron functions. The one-electron wave functions were obtained in the average level scheme by applying the GRASP2K package [21] with a modified RSCF component from the GRASP92 program [22]. The ASF mixing coefficients, and further relativistic and QED corrections, were solved using the RELCI extension [23] of GRASP. The corrections included transverse Breit interaction, mass shift, contributions from self-energy, and vacuum polarization as implemented in RELCI [23]. From the corrections Breit interaction and self-energy are the most important and need to be included for obtaining correct transition energies at this energy region, as discussed, e.g., in Ref. [24].

All calculations included only orbitals that are occupied in the ground state of $\mathrm{Xe}$. The ground state and singly ionized states $L_{1}, L_{2}$, and $L_{3}$ were calculated within a single configuration scheme. The final states of the $L M X$ Auger electron spectrum included all states obtainable from $M M$, $M N$, and $M O$ double-hole configurations. To obtain the correct lifetime broadenings of the $L$ ionized states, Auger decays to all doubly ionized states $M X, N X$, and $O O$, where $X=M, N, O$ were calculated and summed with the widths arising from the $L X$ fluorescence decays. In addition, because the considered double-core-hole states can decay further, Auger decays to all possible triply ionic states $M M M, M N N$, $M N O$, and $N N N$ were calculated. The total lifetime broadening of the presented $L M X$ Auger electron lines are thus defined by the sum of the broadenings of the initial $L$-hole state and final $M X$ double-hole state.

The Auger and fluorescence decay transition matrix elements were calculated utilizing the Auger and Reos components of the RATIP package [25], respectively. The photoionization cross sections were calculated using the Photo component of RATIP [25]. Auger electron emission from $2 s_{1 / 2}^{-1}$ and $2 p_{1 / 2}^{-1}$ states is always isotropic, but generally not from $2 p_{3 / 2}^{-1}$ states. Orientation of electron detection parallel to the polarization vector of the linearly polarized incoming photons was accounted for by calculating the degree of alignment of the $2 p_{3 / 2}^{-1}$ ionized state using the Photo component. The alignment parameter allows defining the angular distribution of Auger electrons [26]. The effect of angular anisotropy was, however, found to be small in the present case. While the normal Auger lines individually show an almost isotropic angular distribution, in the resonant Auger case angular anisotropy is present in individual lines, but when the lines are convolved with final-state-lifetime broadenings, the effects smear out almost completely.

\section{RESULTS AND DISCUSSION}

We will describe the theoretical and experimental results in two separate sections, and, namely, above-threshold data, i.e., normal Auger spectra, and below-threshold data, i.e., resonant Auger spectra. In the first section we shall discuss the experimental and theoretical results of the normal Auger spectra over a wide kinetic-energy range of $2500-4700 \mathrm{eV}$.
Since these spectra contain approximately 400 diagram lines we will give the assignment only on the basis of groups, such as, e.g., $L_{1} M_{2,3} M_{4,5}$ or $L_{3} M_{4,5} N_{4,5}$. However, a detailed assignment of the individual lines, including their theoretical energy positions and intensities, is presented in a table in the Supplemental Material [27]. In the second section we discuss the resonant $L M_{4,5} M_{4,5}$ Auger transitions and give their assignment. Due to the much narrower energy ranges of these spectra with widths of $45-65 \mathrm{eV}$ we assign in this section each individual Auger transition.

\section{A. Normal Auger spectra}

We obtained normal Auger spectra in a large kinetic-energy range after photoionization of each of the three edges $L_{1}, L_{2}$, and $L_{3}$. We believe that our detailed spectra, measured under state-of-the-art conditions, could become a reference point for future work on similar subjects.

In Fig. 1 we show an overview of Auger spectra measured approximately $12 \mathrm{eV}$ above $L_{3}$ (a), $L_{2}$ (b), and $L_{1}$ (c) photoionization thresholds. The corresponding photon energies are 4799.2, 5118.6, and $5465.3 \mathrm{eV}$. These photon energies are chosen so that the shake-up satellites in the corresponding photoelectron spectra cannot be excited. As a consequence of the chosen photon energies, (a) shows $L_{3} M M, L_{3} M N$, and $L_{3} N N$ Auger transitions only, while (b) contains $L_{3} M M, L_{3} M N$, and $L_{3} N N$ as well as $L_{2} M M, L_{2} M N$, and $L_{2} N N$ transitions. In addition to this (c) contains $L_{1} M M$ and $L_{1} M N$ transitions while the $L_{1} N N$ transitions are located at higher kinetic energies. The Auger transitions are assigned only in the spectrum in which they occur for the first time in terms of increasing photon energy. The black lines identify the Auger final states, while the green lines identify photoemission lines due to the ionization of $\mathrm{Xe} 3 s^{-1}, 3 p^{-1}, 3 d^{-1}, 4 s^{-1}$, and $4 p^{-1}$ levels with the binding energies of $1148.7 \mathrm{eV}\left(3 s_{1 / 2}^{-1}\right), 1002.1 \mathrm{eV}\left(3 p_{1 / 2}^{-1}\right)$, $940.6 \mathrm{eV}\left(3 p_{3 / 2}^{-1}\right), 689.0 \mathrm{eV}\left(3 d_{3 / 2}^{-1}\right), 676.4 \mathrm{eV}\left(3 d_{5 / 2}^{-1}\right), 213.2 \mathrm{eV}$ $\left(4 s_{1 / 2}^{-1}\right), 146.7 \mathrm{eV}\left(4 p_{1 / 2}^{-1}\right)$, and $145.5 \mathrm{eV}\left(4 p_{3 / 2}^{-1}\right)$ [28]. Among these lines, $\mathrm{Xe} 4 s^{-1}$ and $4 p^{-1}$ are present only in Fig. 1(a), and Xe $3 d^{-1}$ in Figs. 1(a) and 1(b), while Xe $3 s^{-1}$ and $3 p^{-1}$ are present in all spectra. The intensities for all normal Auger spectra are given in arbitrary units by scaling the maximum of the $L_{3} M_{4,5} M_{4,5}$ transitions $\left(E_{\mathrm{kin}}=3386.6 \mathrm{eV}\right)$ in the spectrum measured at $5465.3 \mathrm{eV}$ to 100 , see Fig. 1(c). The higher intensities of this maximum in the spectra shown in panels 1(b) and (a), namely, 125 and 179, respectively, are due to the increase of the $2 p_{3 / 2}^{-1}$ photoionization cross section with decreasing photon energy. For the scaling of the spectra in Figs. 1(a) and 1(b) the calculated energy dependence of the $2 p_{3 / 2}^{-1}$ photoionization cross section is used. The photon-energy dependence of the photoionization cross sections also partially explains changes in the relative intensities between Figs. 1(b) and 1(c). As an example, the ratio of the peak intensities of the $L_{2} M_{4,5} M_{4,5}$ and $L_{3} M_{4,5} M_{4,5}$ transitions decreases from about 5:10 in (b) to about 4:10 in (c) which is due to the fact that the $2 p_{1 / 2}^{-1}$ photoionization cross section drops between the photon energies 5118.6 and $5465.3 \mathrm{eV}$ faster than the $2 p_{3 / 2}^{-1}$ photoionization cross section.

In Fig. 2 we show a direct comparison between $L M M$ Auger spectra recorded at $h v=5465.3 \mathrm{eV}$ (black) and the 


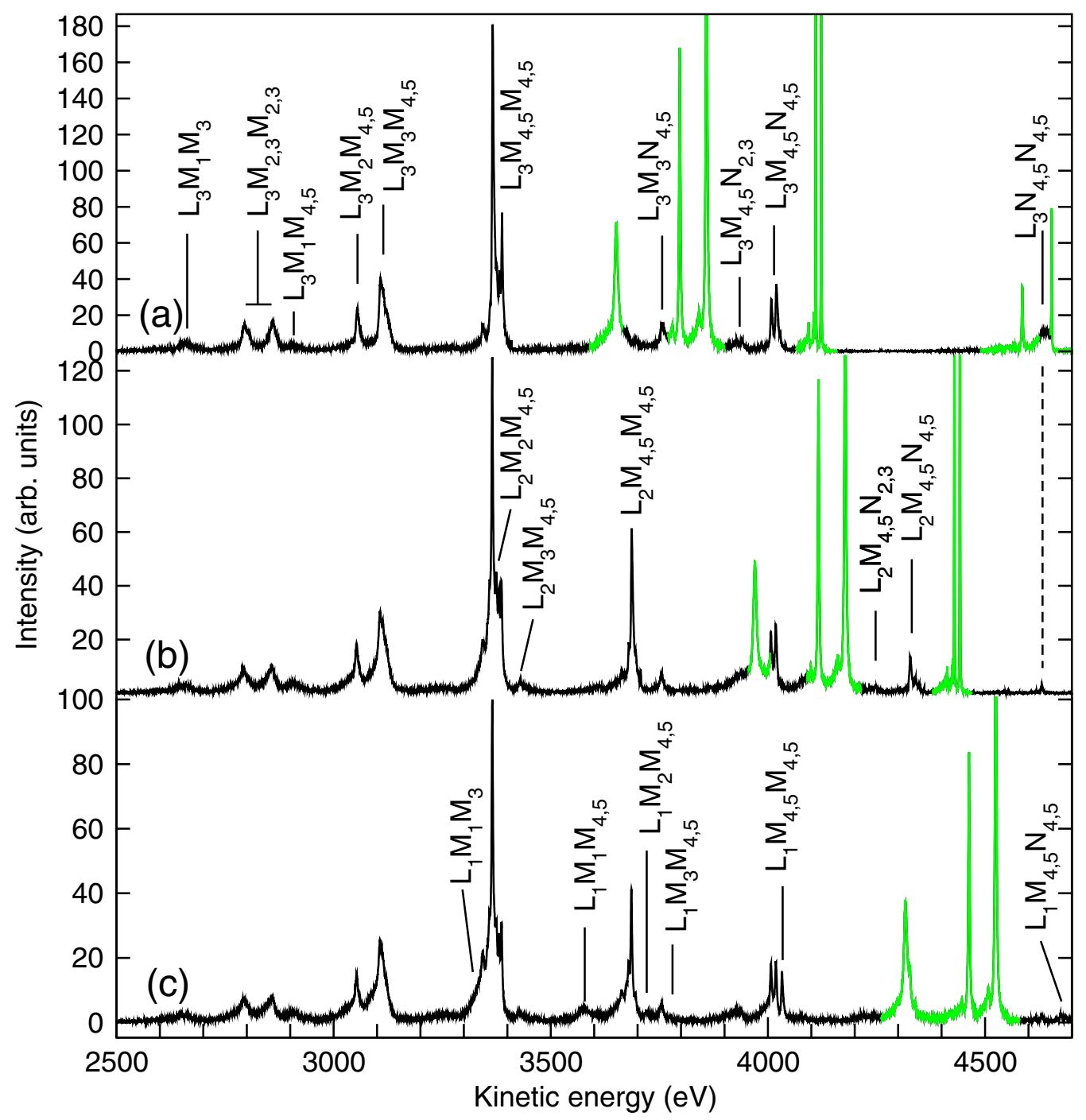

FIG. 1. Overview spectra of Xe $L M M, L M N$, and $L N N$ Auger transitions in the kinetic-energy range from 2500 to $4700 \mathrm{eV}$. The spectra (a-c) are measured at photon energies of 4799.2, 5118.6, and $5465.3 \mathrm{eV}$, i.e., approximately $12 \mathrm{eV}$ above the $L_{3}, L_{2}$, and $L_{1}$ thresholds, respectively. The black parts of the spectra indicate Auger transitions and the green parts contributions of the overlapping photoelectron lines $\mathrm{Xe} 3 s^{-1}, 3 p^{-1}, 3 d^{-1}, 4 s^{-1}$, and $4 p^{-1}$. For more details, see text.

corresponding theoretical results (red). The spectrum shows contributions originating from the $L_{3}, L_{2}$, and $L_{1}$ core holes. As discussed above, the intensities are scaled so that the experimental and theoretical maxima of the most intense peak at a kinetic energy of $3365 \mathrm{eV}$ are equal to 100 (in arb. units). The purpose of the figure is to illustrate the excellent agreement between the experimental and the calculated spectrum over a wide energy range of $1500 \mathrm{eV}$, both in terms of relative intensities and widths of the spectral structures. A more careful comparison of the experimental spectrum with the theoretical results shows that for each spectral feature there is an excellent agreement on the higher-kinetic-energy side while on the lower-kinetic-energy side (typically $25-30 \mathrm{eV}$ below the peak positions) obviously intensity is not reproduced in the theoretical spectrum. This misfit can readily be explained with transitions caused by shake processes, mainly during the ionization process and partially also during the Auger decay. Such processes are not taken into account in the present theoretical studies. Clear evidence for contributions caused by shake processes during the ionization can be observed best by comparing the pure $L_{3}$ Auger decays in Figs. 1(a) and 1(b) since these processes contribute only to the spectrum in (b) due to the higher photon energy.

The detailed results of the calculations including the energy positions, intensities, and assignments of the individual Auger transitions are given in the table in the Supplemental Material [27]. As one of the main results of the calculations we obtained that the $M M, M N$, and $N N$ double-core-hole states are described best with $j j$ coupling, i.e., by $\left(n l_{j}^{-1} n^{\prime} l_{j^{\prime}}^{\prime-1}\right)_{J}$, with first CFS mixing coefficients $\left|c_{i}\right|^{2}$ which are typically larger than 0.9; see the table in the Supplemental Material [27].

The Auger spectra displayed in Figs. 1 and 2 are rather complex; thus we will perform the spectral assignment according to the following guidelines: We will subdivide the spectra into several regions, each one of those corresponding to one particular group of Auger transitions and, therefore, 


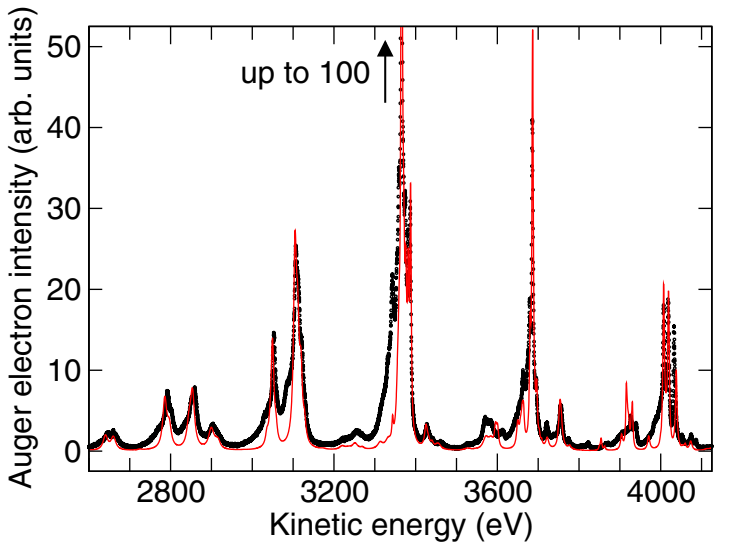

FIG. 2. Comparison of the experimental spectrum recorded at $h v=5465.3 \mathrm{eV}$ [black data points, same as spectrum (c) in Fig. 1] with the theoretical results (red line). The intensities are scaled so that the experimental and theoretical maxima at a kinetic energy of $3365 \mathrm{eV}$ are equal to 100 . For more details, see text.

one binding-energy region for the Auger final states (i.e., $\mathrm{Xe} \rightarrow \mathrm{Xe}^{2+}$ energy difference). The binding-energy scale is derived by subtracting for the $L_{3}$ spectrum the Auger kinetic energy from the ionization potential of $4686 \mathrm{eV}$. Note that PCI effects in the order of $1 \mathrm{eV}$ can additionally apply; see below. Using the binding-energy scale we are able to compare final states with the same one-electron configuration, but deriving from different core-hole-filling processes. We will show these spectral regions in order of increasing Auger binding energy in Figs. 3-7. In these figures, the color code used for the different subspectra and vertical bar diagrams is identical and it will—due to reasons of brevity — be discussed in detail only in connection with Fig. 3. For the other figures only peculiarities will be mentioned.

In Fig. 3 we show the $L_{3} M_{4,5} N_{4,5}$ and $L_{3} M_{4,5} N_{2,3}$ (a), $L_{2} M_{4,5} N_{4,5}$ and $L_{2} M_{4,5} N_{2,3}$ (b), and $L_{1} M_{4,5} M_{4,5}$ and $L_{1} M_{4,5} N_{2,3}$ (c) Auger transitions on the experimental bindingenergy scale of the $M_{4,5} N_{4,5}$ and $M_{4,5} N_{2,3}$ final states (upper axis). The spectra are aligned in binding energy; therefore three different kinetic-energy scales are shown.

In Fig. 3-7, the red lines through the data points represent the results of the calculations and the solid bars the relative intensities and positions of the Auger transitions. In figures 3 and 4 the theoretical spectra for $L_{1}$ (a) and $L_{3}$ (c) are shifted by $1 \mathrm{eV}$ to higher and $5 \mathrm{eV}$ to lower kinetic energies, respectively, in order to account for inaccuracies in calculating the $L_{1}, L_{2}$, and $L_{3}$ binding energies. In this way we aligned in the present case the transition to the $M_{4,5} N_{4,5}$ and $M_{4,5} N_{2,3}$ final states to the same binding energy. In all spectra $(\mathrm{a}-\mathrm{c})$ the black bars indicate the transitions to the final states under discussion, i.e., in the present case the $M_{4,5} N_{4,5}$ and $M_{4,5} N_{2,3}$ final states. Normally, in Figs. 3-7 the black subspectra in the panels $(b, c)$ indicate the theoretical contributions originating from Auger transitions starting at the $L_{2}$ and $L_{1}$ core hole, respectively. In the present case of Fig. 3, however, there is no black subspectrum visible in (b) since there is a perfect overlap with the red subspectrum. The blue subspectrum and blue bars in (b,c) indicate the contributions caused by the $L_{3}$ core hole, while the orange subspectrum and the orange bars (c) indicate

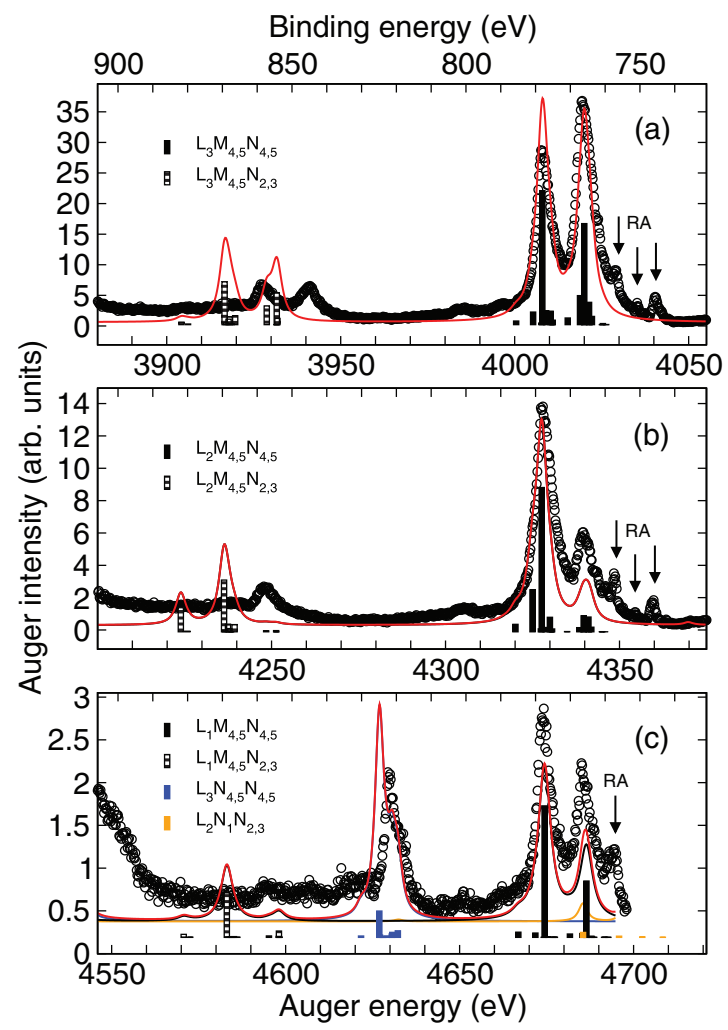

FIG. 3. Comparison of the experimental (data points) and theoretical results (red line) for the $L_{3} M_{4,5} N_{4,5}$ and $L_{3} M_{4,5} N_{2,3}$ (a), $L_{2} M_{4,5} N_{4,5}$ and $L_{2} M_{4,5} N_{2,3}$ (b), and $L_{1} M_{4,5} N_{4,5}, L_{1} M_{4,5} N_{2,3}$, $L_{2} N_{1} N_{2,3}$, and $L_{3} N_{4,5} N_{4,5}$ (c) Auger transitions on the experimental binding-energy scale of the $M_{4,5} N_{4,5}$ and $M_{4,5} N_{2,3}$ final states (upper axis). RA indicates contributions from resonant Auger transitions. For details, see text.

the contributions caused by the $L_{2}$ core hole. The missing of blue bars and a blue subspectrum in Fig. 3(b) indicate that such contributions are absent in the discussed binding-energy region, contrary to all other binding-energy regions discussed below. The subspectra in (c) are only poorly visible since they overlap strongly with each other. However, it is clear that the group of peaks around $4630 \mathrm{eV}$ (blue bars) do not stem from $L_{1}$ decay but from $L_{3}$ decay. Here the slight shift of the theoretical data by approximately $5 \mathrm{eV}$ as compared to the experimental data is due to the fact that the theoretical curve is adjusted to fit the experimental $L_{1}$ transitions and reflects the inaccuracies in calculating the $L$ core holes. Note that the binding-energy scale only holds for the doubly ionic final states indicated by black vertical bars.

Contrary to the $L_{3} M_{4,5} N_{4,5}$ transitions, where the misfit between experiment and theory originates from inaccuracies in calculating the initial states, the disagreement between experiment and theory for the $L M_{4,5} N_{2,3}$ transitions in Figs. 3(a)-3(c) is due to inaccuracies in the final states. The Auger transitions at a binding energy of 791 and $803 \mathrm{eV}$ visible in spectra $(\mathrm{a}-\mathrm{c})$ are also not reproduced by theory. Since the Auger spectra are measured at excitation energies below the first satellites in the photoelectron spectrum, these spectral features can be assigned to $5 p \rightarrow 6 p$ shake-up transitions during the Auger decay [17]. These assignments are based 


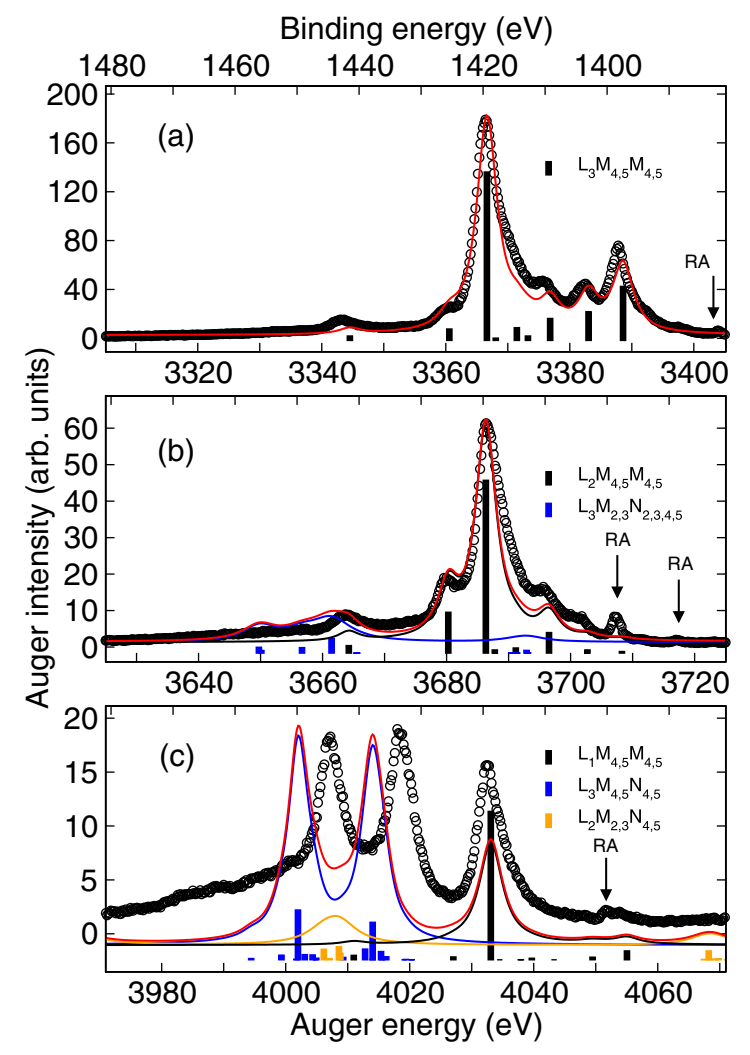

FIG. 4. Comparison of the experimental (data points) and theoretical results (red line) for the $L_{3} M_{4,5} M_{4,5}$ (a), $L_{2} M_{4,5} M_{4,5}$ and $L_{2} M_{2,3} N_{2,3,4,5}$ (b), and $L_{1} M_{4,5} M_{4,5}, L_{2} M_{2,3} N_{4,5}$, and $L_{3} M_{4,5} N_{4,5}$ (c) Auger transitions on the experimental binding-energy scale of the $M_{4,5} M_{4,5}$ final states (upper axis). RA indicates contributions from resonant Auger transitions. For details, see text.

on the fact that the kinetic energies of these two lines are approximately $23 \mathrm{eV}$ below those of the main lines at binding energies of 768 and $780 \mathrm{eV}$, which agrees well with the excitation energies of $22-25 \mathrm{eV}$ for the $5 p^{6} \rightarrow 5 p^{5} 6 p$ transition in the $Z+2$ atom Ba III [20].

The two-dimensional (2D) maps in our previous work [13] clearly show that the population of the final states of the resonant Auger decay extends to excitation energies above the ionization threshold. Their contributions in the present spectra are indicated by vertical arrows labeled with RA and caused by a recapture of the continuum photoelectron to a discrete state during the Auger decay [11]. In principle such resonant Auger contributions should be visible in all Auger transitions measured subsequent to photoionization directly above the corresponding $L$ threshold. They are, however, identified only for the $L M_{4,5} N_{4,5}$ transitions and the $L M_{4,5} M_{4,5}$ transitions; see below. This finding can readily be understood based on linewidth arguments. The linewidth of the normal Auger transitions is given by the $L$ core-hole lifetime $(2.82 \mathrm{eV}$ for $\mathrm{Xe}$ $2 p_{3 / 2}^{-1}, 3.04 \mathrm{eV}$ for Xe $2 p_{1 / 2}^{-1}$, and $3.35 \mathrm{eV}$ for Xe $2 s_{1 / 2}^{-1} \mathrm{eV}$ [29]) as well as the lifetime of the final states and the analyzer resolution $(0.5 \mathrm{eV})$. Contrary to this the linewidth of the resonant Auger transitions is, in the case of resonant Raman conditions (i.e., when the photon bandwidth is considerably smaller than the lifetime broadening of the core-excited state),

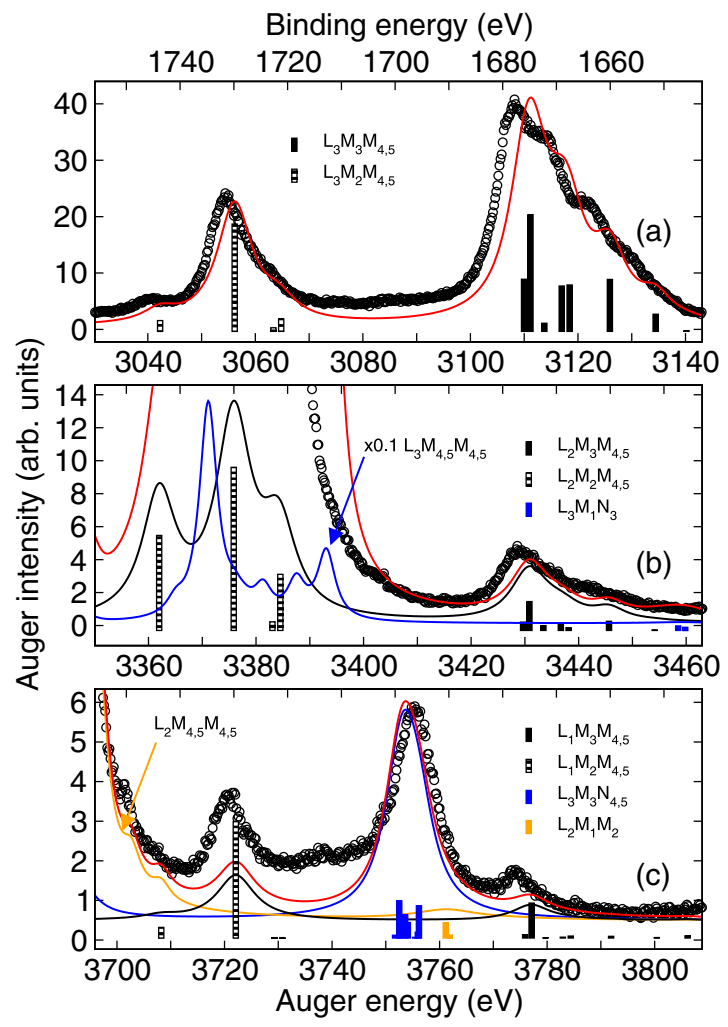

FIG. 5. Comparison of the experimental (data points) and theoretical results (red line) for the $L_{3} M_{2,3} M_{4,5}$ (a), $L_{2} M_{2,3} M_{4,5}$, $L_{3} M_{4,5} M_{4,5}$, and $L_{3} M_{1} N_{3}$ (b), and $L_{1} M_{2,3} M_{4,5}, L_{2} M_{1} M_{2}$, and $L_{3} M_{3} N_{4,5}$ (c) Auger transitions on the experimental binding-energy scale of the $M_{2,3} M_{4,5}$ final states (upper axis). For details, see text.

dictated by the photon bandwidth $(0.6 \mathrm{eV})$, the lifetime widths of the final states, and the analyzer resolution $(0.5 \mathrm{eV})$. Because of this the resonant Auger spectra of transitions to long-lived final states are narrower and more pronounced than the normal Auger spectra.

In the present theoretical results, see red lines in Figs. 3-7, the lifetime broadening of the final states is taken into account and leads to an overall good agreement between experiment and theory. However, the values for the lifetime broadenings of the two-hole final states can be considered only as semiquantitative since they partially show a significant dependence on the two-hole configurations included in the calculations. This shows that for accurate numbers of the double-core-hole lifetimes more dedicated calculations are required. Despite this finding it is unquestionable that the final states $M_{4,5} N$ and $M_{4,5} M_{4,5}$ exhibit the smallest lifetime broadenings of all two-hole final states. This explains the presence of the resonant Auger contributions in the $L M_{4,5} N_{4,5}$ transitions and the $L M_{4,5} M_{4,5}$ transitions. For the Auger transitions to final states other than $M_{4,5} M_{4,5}$ and $M_{4,5} N$ the lifetime widths of the final states are much larger, and they are the dominant contributions to the total widths of the normal Auger and the resonant Auger lines. Because of this the resonant Auger lines are not significantly narrower and more pronounced than the normal Auger lines, explaining their apparent absence in the spectra. In addition, the calculations fully support that the observed width of Auger electron lines may not represent the 


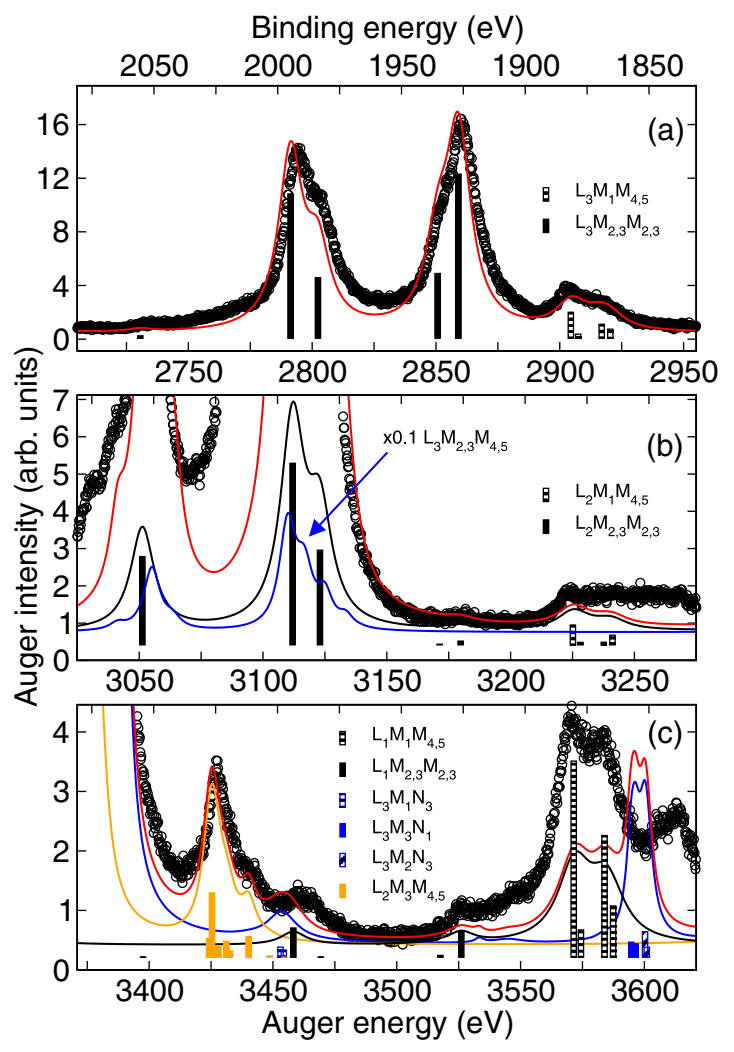

FIG. 6. Comparison of the experimental (data points) and theoretical results (red line) for the $L_{3} M_{1} M_{4,5}$ and $L_{3} M_{2,3} M_{2,3}$ (a), $L_{2} M_{1} M_{4,5}, L_{2} M_{2,3} M_{2,3}$, and $L_{3} M_{2,3} M_{4,5}$ (b), and the $L_{1} M_{1} M_{4,5}, L_{1} M_{2,3} M_{2,3}, L_{2} M_{3} M_{4,5}, L_{3} M_{1} N_{3}, L_{3} M_{3} N_{1}$, and $L_{3} M_{2} N_{3}$ (c) Auger transitions on the experimental binding-energy scale of the $M_{1} M_{4,5}$ and $M_{2,3} M_{2,3}$ final states (upper axis). For details, see text.

lifetime of the initial state, but instead the width of final states that decay further. This is, e.g., visualized in the spectrum of Fig. 1(a), which shows Auger lines of considerably different widths, even though they all arise from the same initial state. To illustrate, $L M_{4,5} M_{4,5}$ lines appear narrowest because $M_{4,5} M_{4,5}$ double-hole states can decay only to $M_{4,5} N N$ triple hole states. When the two holes are deeper, first Coster-Kronig decay to $M M N$ final states become accessible and finally in the case of the $M_{1} M_{1}$ double-hole state a decay to a super Coster-Kronig-type $M_{1} M_{1}-M M M$ channel is open.

Finally, we want to point out that the transitions indicated with black subspectra and black vertical bars are significantly influenced by the postcollision interaction (PCI) effect. So, for example, we can explain the discrepancy between the experimental and theoretical intensity ratios of the $L_{3} M_{4,5} M_{4,5}$ Auger transitions in Fig. 3(a) with the influence of PCI. This is supported by the finding that after ionization with much higher photon energies the theoretical results much better match the experimental spectrum; see Fig. 4(c).

In Fig. 4 we show the $L_{3} M_{4,5} M_{4,5}$ (a), $L_{2} M_{4,5} M_{4,5}$ (b), and $L_{1} M_{4,5} M_{4,5}$ (c) Auger transitions on the experimental binding-energy scale of the $M_{4,5} M_{4,5}$ final states (upper axis). As stated above, in Figs. 3-7 identical color codes of the different subspectra and vertical bars are used; they are discussed in connection with Fig. 3. Obviously, the $L M_{4,5} M_{4,5}$

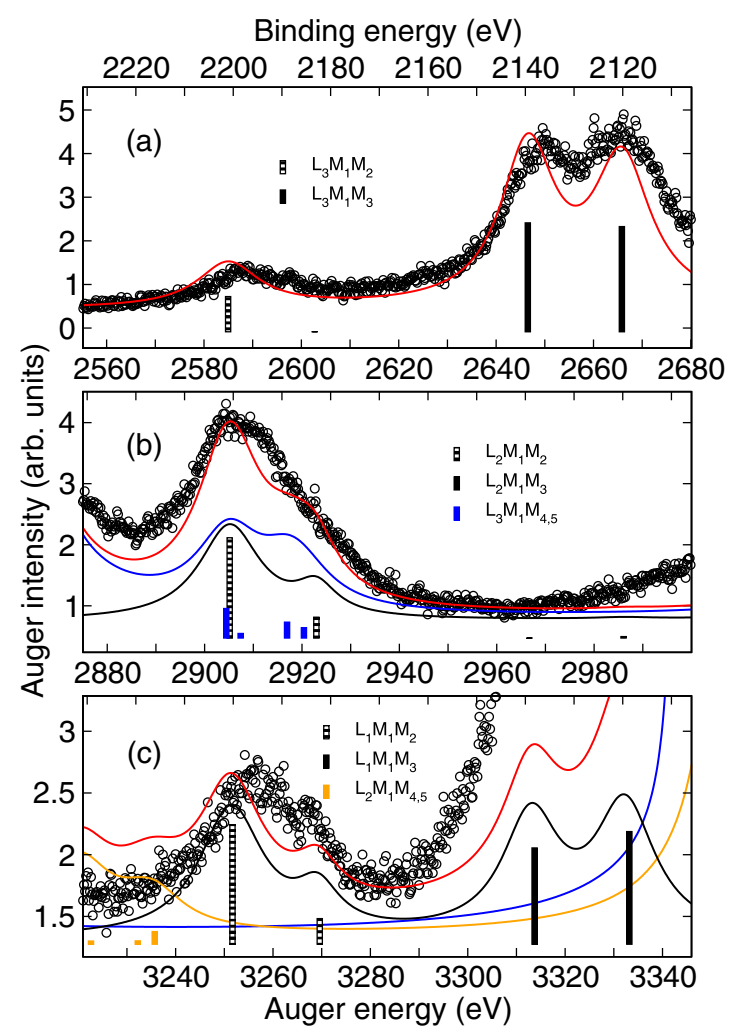

FIG. 7. Comparison of the experimental (data points) and theoretical results (red line) for the $L_{3} M_{1} M_{2}$ and $L_{3} M_{1} M_{3}$ (a), $L_{2} M_{1} M_{2}$, $L_{2} M_{1} M_{3}$, and $L_{3} M_{1} M_{4,5}(\mathrm{~b})$, and the $L_{1} M_{1} M_{2}, L_{1} M_{1} M_{3}, L_{2} M_{3} M_{4,5}$, and $L_{2} M_{1} M_{4,5}$ (c) Auger transitions on the experimental bindingenergy scale of the $M_{1} M_{2}$ and $M_{1} M_{3}$ final states (upper axis). For details, see text.

Auger transitions are described well by the theory indicated with the red lines. In Fig. 4(c) the intense transition at a kinetic energy of $4032 \mathrm{eV}$ is, according to the calculations, formed by a single Auger transition. Nevertheless, the line shape exhibits a significant asymmetry which is caused by the PCI effect due to the slow photoelectron with a kinetic energy of approximately $12 \mathrm{eV}$; for further discussion, see below.

It is interesting to note that in Fig. 4(c) the contributions of the $L_{3} M_{4,5} N_{4,5}$ Auger transitions indicated by the blue subspectrum and the blue vertical bars accidentally overlap with the $L_{1} M_{4,5} M_{4,5}$ Auger transitions, which are in the main focus of this energy region. Moreover, both groups of lines have similar linewidths and intensities, showing that the Auger spectra of the different $L$ core holes overlap and cannot be distinguished easily based on the spectral features. Superficially, it is surprising that the $L_{3} M_{4,5} N_{4,5}$ Auger transitions are more intense than the $L_{1} M_{4,5} M_{4,5}$ Auger transitions, since Auger intensities are governed by overlap arguments so that $M_{4,5} M_{4,5}$ Auger transitions should be preferred. As an example, the KLL:KLM:KMM Auger intensity ratios in argon are about 0.87:0.12:0.01 [30]. However, a closer inspection of the theoretical results clearly shows that this expectation also holds for the present case. In particular, for all $L$ holes the $M_{4,5} M_{4,5}: M_{4,5} N_{4,5}$ Auger intensity ratio is $\cong 0.8: 0.2$. As a consequence, the higher intensity of the 
$L_{3} M_{4,5} N_{4,5}$ Auger transitions as compared to the $L_{1} M_{4,5} M_{4,5}$ Auger transitions is due to (i) the approximately 4 times higher calculated ionization probability of the $2 p_{3 / 2}^{-1}$ state than of the $2 s_{1 / 2}^{-1}$ state at the photon energy of $5456.3 \mathrm{eV}$ and (ii) a total Auger rate to the $M_{4,5} M_{4,5}$ and $M_{4,5} N_{4,5}$ final states, which is lower by a factor of 3 for the $2 s^{-1}$ core hole as compared to the $2 p^{-1}$ core holes.

We also want to point out that the misfit of the energy positions of the $L_{3} M_{4,5} N_{4,5}$ Auger transitions in Fig. 4(c) is due to an adjustment of the curve to the $L_{1}$ transitions; i.e., it reflects the inaccuracies in the $L$-core-hole binding energies but not of the two-hole final states. A comparison of the low-kinetic-energy tail (3980-4000 eV) of the $L_{3} M_{4,5} N_{4,5}$ Auger transitions in Fig. 3(a) measured at a photon energy of $4799.2 \mathrm{eV}$ and in Fig. 4(c) measured at $5456.3 \mathrm{eV}$ shows in the latter spectrum a significant increase of the intensity. This increase is due to the Auger decays of the Xe $2 p_{3 / 2}^{-1}$ photoelectron satellites. Contributions caused by resonant Auger related to the $L_{3} M_{4,5} M_{4,5}$ Auger transitions are indicated by vertical bars. These types of resonant Auger transitions leading the $3 d^{-2} n l$ final states will be discussed in detail further below. Note that the $M_{4,5} M_{4,5}$ final states in this energy region are the corresponding parent states.

Figure 1(c) demonstrates that the $L_{3} M_{4,5} M_{4,5}$ Auger transition at $E_{\text {kin }}=3386.6 \mathrm{eV}$ which is labeled in panel (a) is the most intense one in the energy region discussed in the present publication. With the exception of our own publication on all three $L M_{4,5} M_{4,5}$ resonant Auger transitions [13], it is to the best of our knowledge the only region that has been discussed in some detail in the literature [31,32]. Both publications focus on the resonant Auger decay of the $L_{3}$ core hole to the final states related to the $\left(3 d_{3 / 2}^{-1} 3 d_{5 / 2}^{-1}\right)_{4}$ parent state, and label this state $M_{4} M_{5}\left({ }^{1} G_{4}\right)$, i.e., they use $L S$ coupling. However, both publications also report the $\mathrm{Xe} 2 p_{3 / 2}^{-1} \rightarrow\left(3 d_{3 / 2}^{-1} 3 d_{5 / 2}^{-1}\right)_{4}$ diagram line. In addition, Brown et al. [31] report a theoretical Auger spectrum for the different diagram lines that matches, with respect to energy positions and Auger intensities, quite well with the present results shown in Fig. 4(a). The main difference from the present results is that Brown et al. use $L S$ terms to describe the $3 d^{-2}$ final states, although the present calculations show that $j j$ terms are very well suited to describe these two-hole states. This can be seen by the leading mixing coefficients $\left|c_{1}\right|^{2}$ presented in the Supplemental Material [27], which are almost all very close to 1 . This finding is in line with the observation that the energy splittings between the $3 d^{-2}$ final states labeled by Brown et al. as ${ }^{3} P_{2,1,0}$ and ${ }^{3} F_{4,3,2}$ do not obey the Landé interval rule which is strictly valid in $L S$ coupling. In particular, for the ${ }^{3} F_{4,3,2}$ states even the observed sequence of states does not follow the one expected in pure $L S$ coupling. But we also want to point out that the $L S$ terms are at least for part of the states not completely meaningless. This can be shown by describing the present $\left(3 d_{j_{1}}^{-1} 3 d_{j_{2}}^{-1}\right)_{J}$ states or $\left(3 d_{j}^{-2}\right)_{J}$ states in case of $j_{1}$ equal to $j_{2}$ in a ${ }^{2 S+1} L_{J}$ basis. For this purpose we used

$$
\left|\left(l_{1} s_{1}\right) j_{1},\left(l_{2} s_{2}\right) j_{2} ; J M\right\rangle=\sum_{L, S} T_{L S ; j_{1} j_{2}}\left|\left(l_{1} l_{2}\right) L,\left(s_{1} s_{2}\right) S ; J M\right\rangle
$$

with $T_{L S ; j_{1} j_{2}}$ in this expression being based on $9 j$ symbols, namely [33],

$$
\begin{aligned}
T_{L S ; j_{1} j_{2}}= & \sqrt{(2 L+1)(2 S+1)\left(2 j_{1}+1\right)\left(2 j_{2}+1\right)} \\
& \times\left\{\begin{array}{ccc}
l_{1} & l_{2} & L \\
s_{1} & s_{2} & S \\
j_{1} & j_{2} & J
\end{array}\right\} .
\end{aligned}
$$

Using these equations it can readily be found that the double-core-hole state $\left(3 d_{3 / 2}^{-1} 3 d_{5 / 2}^{-1}\right)_{4}$ shows $80 \%{ }^{1} G_{4}$ and $20 \%{ }^{3} F_{4}$ character while the state $\left(3 d_{5 / 2}^{-2}\right)_{4}$ possess $20 \%$ ${ }^{1} G_{4}$ and $80 \%{ }^{3} F_{4}$ character, in reasonable agreement with the assignment of Brown et al. [31]. Moreover, the states $\left(3 d_{3 / 2}^{-1} 3 d_{5 / 2}^{-1}\right)_{3}$ and $\left(3 d_{3 / 2}^{-1} 3 d_{5 / 2}^{-1}\right)_{1}$ show $100 \%{ }^{3} F_{3}$ and ${ }^{1} P_{1}$ character, respectively. Contrary to this, significant mixing is found for the double-core-hole states with $J=2$ and 0 . The observation that $L S$ coupling is a good approximation for the states with $J=4,3$, and 1 is due to the fact that both holes are in the $3 d$ shell so that the Pauli principle excludes some ${ }^{2 S+1} L_{J}$ states such as ${ }^{3} G_{5,4,3}$, which are allowed in $n d^{-1} m d^{-1}$ configurations. As a consequence, mixing of ${ }^{2 S+1} L_{J}$ states with the same value of $J$ is substantially suppressed, leading in the case of two holes in the same $d$ shell to simpler transformations between $j j$ and $L S$ states.

In Fig. 5 we show the $L_{3} M_{2,3} M_{4,5}$ (a), $L_{2} M_{2,3} M_{4,5}$ (b), and $L_{1} M_{2,3} M_{4,5}$ (c) Auger transitions on the experimental binding-energy scale of the $M_{2,3} M_{4,5}$ final states (upper axis). In 5(b) the blue subspectrum is multiplied by the factor 0.1 in order to show the $L_{3} M_{4,5} M_{4,5}$ Auger transitions dominant in this energy region. In the present case as well as in Figs. 6 and 7, see below, the theoretical spectra in Figs. 5(a) and 5(b) are shifted by 6 and $5 \mathrm{eV}$ to higher kinetic energies in order to account for inaccuracies in calculating the Auger final states in addition to those for the core-hole states; see above. The $L_{1} M_{3} M_{4,5}$ to $L_{3} M_{3} N_{4,5}$ intensity ratio in Fig. 5(c) is similar to that for the $L_{1} M_{4,5} M_{4,5}$ to $L_{3} M_{4,5} N_{4,5}$ Auger intensity ratio seen in Fig. 4(c); i.e., the latter transitions including $4 d^{-1}$ holes in the final state are more intense. Based on the theoretical results, the explanation of this finding is similar to that for the $L_{1} M_{4,5} M_{4,5}$ to $L_{3} M_{4,5} N_{4,5}$ Auger intensity ratio. First, the calculated ionization probability of the $2 p_{3 / 2}^{-1}$ state is approximately 4 times higher than that of the $2 s_{1 / 2}^{-1}$ state. Second, the total Auger rate to the $M_{4,5} M_{4,5}$ and $M_{4,5} N_{4,5}$ final states is for the $2 s_{1 / 2}^{-1}$ core hole by a factor of 2 lower than for the $2 p_{3 / 2}^{-1}$ core hole. Contrary to this, for each discussed initial core hole the ratio of the Auger rates to the final states $M_{3} M_{4,5}$ to $M_{3} N_{4,5}$ is between $4: 1$ and $6: 1$; i.e., the transition rates to the final states with $3 d^{-1} 4 d^{-1}$ holes are, fully in line with the expectations, considerably smaller than those with $3 d^{-2}$ final states.

In Fig. 6 we show the $L_{3} M_{1} M_{4,5}$ and $L_{3} M_{2,3} M_{2,3}$ (a), $L_{2} M_{1} M_{4,5}$ and $L_{2} M_{2,3} M_{2,3}$ (b), and $L_{1} M_{1} M_{4,5}$ and $L_{1} M_{2,3} M_{2,3}$ (c) Auger transitions on the experimental bindingenergy scale of the $M_{1} M_{4,5}$ and $M_{2,3} M_{2,3}$ final states (upper axis). In Fig. 6(a) a weak peak is visible approximately $25 \mathrm{eV}$ below the $L_{3} M_{2,3} M_{2,3}$ transition $2795 \mathrm{eV}$. As discussed above the peak at $2770 \mathrm{eV}$ is probably due to a $L_{3} M_{2,3} M_{2,3}$ transition accompanied by a $5 p \rightarrow 6 p$ shake-up process. Obviously these shake transitions during the Auger decay are very weak 
TABLE I. Experimental, $E_{\text {expt }}$, and theoretical, $E_{\text {th }}$, Auger energies for selected Auger transitions. All energies are given in $\mathrm{eV}$ after eliminating the PCI shift. All values are in addition subject to an uncertainty of $0.7 \mathrm{eV}$ for the entire kinetic-energy scale. For more details, see text.

\begin{tabular}{llc}
\hline \hline \multirow{2}{*}{ Transition } & \multicolumn{2}{c}{ Auger energy } \\
\cline { 2 - 3 } & \multicolumn{1}{c}{$E_{\text {expt }}$} & $E_{\text {th }}$ \\
\hline$L_{3} N_{4,5} N_{4,5}{ }^{a}$ & $4629.6(2) \mathrm{eV}$ & $4631.93 \mathrm{eV}$ \\
$L_{3} M_{4,5} N_{4,5}$ & $4018.2(2) \mathrm{eV}$ & $4019.00 \mathrm{eV}$ \\
& $4006.9(2) \mathrm{eV}$ & $4006.96 \mathrm{eV}$ \\
$L_{3} M_{4,5} M_{4,5}$ & $3386.6(2) \mathrm{eV}$ & $3387.57 \mathrm{eV}$
\end{tabular}

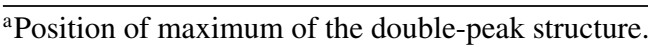

so that they can be observed only in cases of a strong, narrow, and well isolated main peak. In Fig. 6(b) the blue subspectrum indicating the $L_{3}$ Auger transitions is multiplied by the factor 0.1 in order to show the $L_{3} M_{2,3} M_{4,5}$ Auger transitions dominant in this energy region. Finally, in Fig. 7 we show the $L_{3} M_{1} M_{2,3}$ (a), $L_{2} M_{1} M_{2,3}$ (b), and $L_{1} M_{1} M_{2,3}$ (c) Auger transitions on the experimental binding-energy scale of the $M_{1} M_{2,3}$ (upper axis).

Eventually we want to give some values of kinetic energies that can be used for calibration purposes. In this context it is important to note that in the present xenon spectra the kinetic energies of the Auger transitions are significantly photon-energy dependent because of two reasons. First, the postcollision interaction (PCI) effect amounts up to a $1.7 \mathrm{eV}$ $\left(L_{1} M_{4,5} N_{4,5}\right)$ Auger transition in Fig. 3(c), i.e., about 2.5 times the estimated uncertainty of the kinetic-energy scale. Second, an increase of the photon energy across another ionization threshold can open additional Auger channels that overlap with already existing Auger channels. This can shift the maxima of the spectral features as can be seen, e.g., in Fig. 7(b) for the $L_{2} M_{1} M_{3}$ and $L_{3} M_{1} M_{4,5}$ Auger transitions.

To take the two above-described effects into account, we selected a number of well-separated Auger transitions and summarized their Auger energies in Table I. To eliminate the PCI shift on the kinetic energies we simulated the line shapes using the formula given by Armen et al. [34]. Moreover, for this purpose we used the lifetime broadenings of 2.82, 3.04, and $3.35 \mathrm{eV}$ for the thresholds $L_{3}, L_{2}$, and $L_{1}$, respectively [29]. We want to point out that the PCI shift cannot be neglected even several hundred $\mathrm{eV}$ above the respective ionization threshold. For example, at a photon energy of $5465.3 \mathrm{eV}$, i.e., approximately $680 \mathrm{eV}$ above threshold, the PCI shift for the $L_{3} N_{4,5} N_{4,5}$ Auger transition at a kinetic energy of $4630 \mathrm{eV}$ still amounts to $125 \mathrm{meV}$; this PCI effect is due to the short lifetime of the core-ionized state and the high Auger electron energy. In detail, we have selected the $L_{3} M_{4,5} M_{4,5}$ Auger transition with the highest kinetic energy, $2 p_{3 / 2}^{-1} \rightarrow\left(3 d_{5 / 2}^{-2}\right)_{4}$ [see Fig. 4(a)], and the two spectral features caused by the $L_{3} M_{4,5} N_{4,5}$ Auger transitions [see Figs. 4(c), 8(e), and 8(f)], as well as the $L_{3} N_{4,5} N_{4,5}$ Auger transitions [see Fig. 3(c)]. In the latter case we give the absolute maximum of the double-peak structure.

\section{B. Resonant Auger spectra}

We have already published in [13] an overview of the $L M_{4,5} M_{4,5}$ resonant Auger spectra following photoexcitation below all three $L$ edges. There, we focused on the analysis of the partial cross sections created by the most intense resonant Auger transitions, i.e., those that belong to the $2 p^{-1} \rightarrow$ $\left(3 d_{3 / 2}^{-1} 3 d_{5 / 2}^{-1}\right)_{4}$ diagram line. Because of this, the resonant Auger spectra were shown only for the energy region of the $2 p^{-1} \rightarrow$ $\left(3 d_{3 / 2}^{-1} 3 d_{5 / 2}^{-1}\right)_{4} n l$ transitions and we used for the assignment of the final states the one-electron configuration (e.g., $3 d^{-2} 5 d$ for the decay after $L_{2}$ and $L_{3}$ photoexcitation). Moreover, we assigned the $3 d^{-2}$ double-core-hole state $\left(3 d_{3 / 2}^{-1} 3 d_{5 / 2}^{-1}\right)_{4}$ in $L S$ coupling to ${ }^{1} G_{4}$; compare above. Here we implement our results by providing the resonant Auger spectrum over a broader energy range including the weaker transitions and by giving a much more detailed assignment based on $j j$ coupling for the $3 d^{-2}$ parent states. This will also elucidate some intensity ratios of the resonant Auger transitions that look, in a superficial consideration, surprising.

In Fig. 8 we present the resonant Auger spectra measured at $h v=4784.9 \mathrm{eV}$, i.e., top of $\mathrm{Xe} 2 p_{3 / 2} \rightarrow 5 d$ excitation (a), at $h v=4785.9 \mathrm{eV}$ [35], i.e., top of $\mathrm{Xe} 2 p_{3 / 2} \rightarrow 6 d$ excitation (b), at $h v=5105.4 \mathrm{eV}$, i.e., top of $\mathrm{Xe} 2 p_{1 / 2} \rightarrow 5 d$ excitation (c), at $h v=5106.6 \mathrm{eV}$, i.e., top of $\mathrm{Xe} 2 p_{1 / 2} \rightarrow 6 d$ excitation (d), at $h v=5451.37 \mathrm{eV}$, i.e., top of $\mathrm{Xe} 2 s_{1 / 2} \rightarrow 6 p$ excitation (e), and at $h v=5452.77 \mathrm{eV}$, i.e., top of $\mathrm{Xe} 2 s_{1 / 2} \rightarrow 7 p$ excitation (e). Contrary to our previous publication [13], where the spectra were shown on the kinetic-energy scale of the Auger electrons, we present the spectra here on the binding-energy scale so that the Auger final states can be compared directly. As a result of this energy scale, the different $n l$ final states visible in the different spectra form Rydberg series converging towards the corresponding parent state; this is indicated for the $\left(3 d_{3 / 2}^{-2}\right)_{0}$ parent state on the left side of panel (a). For each photon energy, the experimental and theoretical results are given on the binding-energy scale by using black circles and red solid lines, respectively.

For each parent state the energy region of the $3 d^{-2} n l$ final states with $n l=5 d, 6 p, 6 d, 7 p$, and $7 d$ are indicated in turn by black or blue horizontal lines. The solid and dashed vertical lines below each horizontal line indicate the energy positions of the $3 d^{-2} n p$ and $3 d^{-2} n d$ final states belonging to the corresponding parent state, respectively. For the parent states $\left(3 d_{3 / 2}^{-1} 3 d_{5 / 2}^{-1}\right)_{2},\left(3 d_{3 / 2}^{-1} 3 d_{5 / 2}^{-1}\right)_{3},\left(3 d_{5 / 2}^{-2}\right)_{0}$, and $\left(3 d_{3 / 2}^{-2}\right)_{2}$ only part of the final states $3 d^{-2} n d$ are visible and indicated by vertical lines. As can be seen in some energy regions the vertical lines belonging to different parent states almost overlap; i.e., the corresponding spectral feature comprises different resonant Auger transitions. The vertical arrows in $(e, f)$ indicate the $L_{3} M_{4,5} N_{4,5}$ normal Auger transitions overlapping with the $L_{1} M_{4,5} M_{4,5}$ resonant Auger lines.

In Figs. 8(e) and 8(f) we show the results for resonant Auger decay below the $L_{1}$ edge measured at the maximum of the $2 s \rightarrow 6 p$ resonance at $5451.37 \mathrm{eV}$ and at the $2 s \rightarrow 7 p$ resonance at $5452.77 \mathrm{eV}$, respectively. In both spectra the two most intense resonant Auger transitions to the $\left(3 d_{3 / 2}^{-1} 3 d_{5 / 2}^{-1}\right)_{4} 6 p$ and $\left(3 d_{3 / 2}^{-1} 3 d_{5 / 2}^{-1}\right)_{4} 7 p$ final states are observed at binding energies of 1414 and $1417.4 \mathrm{eV}$, respectively. Subsequent to the excitation 


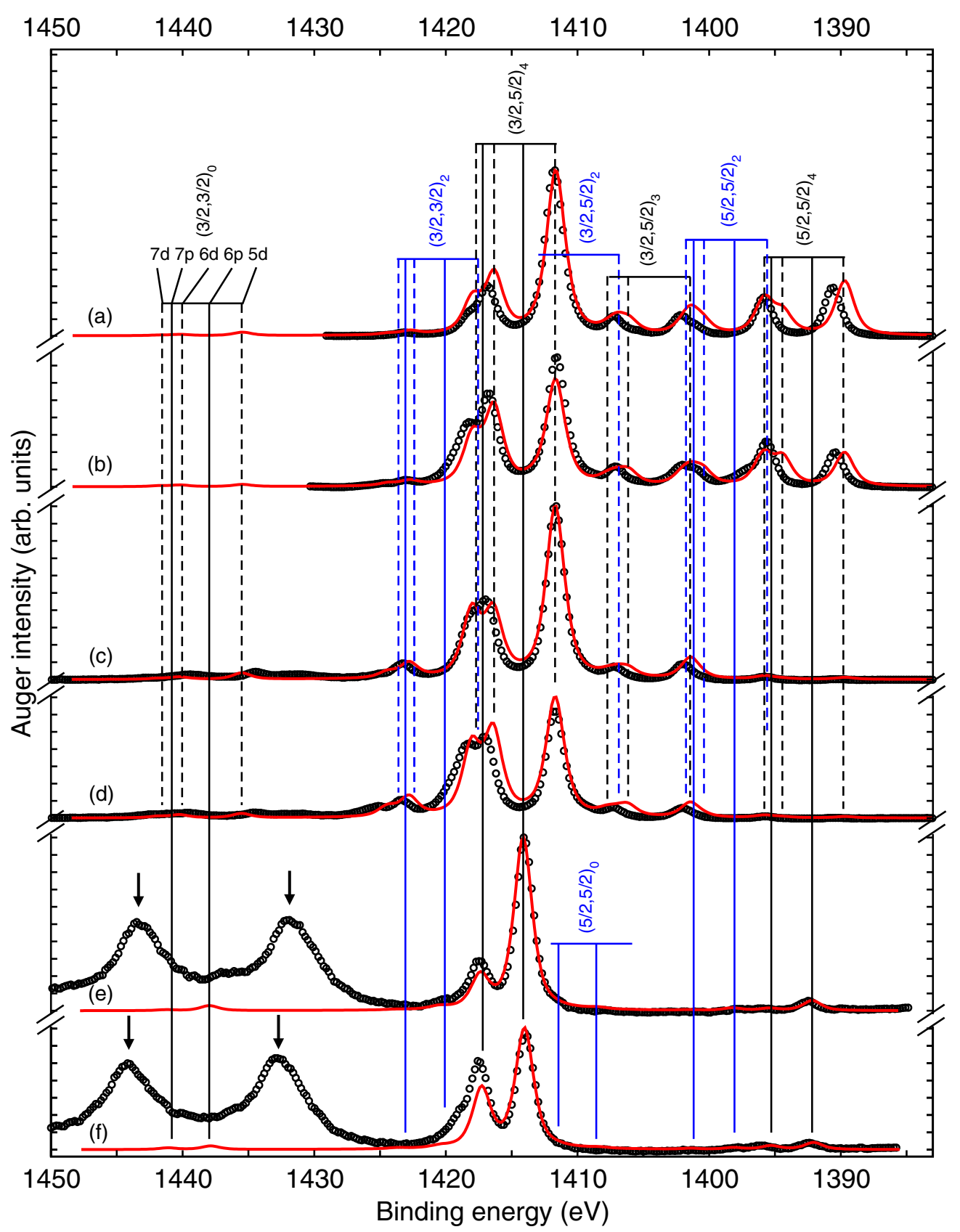

FIG. 8. The $L M_{4,5} M_{4,5}$ resonant Auger spectra measured at $h v=4784.9 \mathrm{eV}$, i.e., top of $\mathrm{Xe} 2 p_{3 / 2} \rightarrow 5 d$ excitation (a), at $h v=4785.9 \mathrm{eV}$, i.e., top of $\mathrm{Xe} 2 p_{3 / 2} \rightarrow 6 d$ excitation (b), at $h v=5105.4 \mathrm{eV}$, i.e., top of $\mathrm{Xe} 2 p_{1 / 2} \rightarrow 5 d$ excitation (c), at $h v=5106.6 \mathrm{eV}$, i.e., top of $\mathrm{Xe} 2 p_{1 / 2} \rightarrow 6 d$ excitation (d), at $h v=5451.37 \mathrm{eV}$, i.e., top of Xe $2 s_{1 / 2} \rightarrow 6 p$ excitation (e), and at $h v=5452.77 \mathrm{eV}$, i.e., top of Xe $2 s_{1 / 2} \rightarrow 7 p$ excitation (e). For each photon energy, the experimental and theoretical results are given on the binding-energy scale by using black circles and red solid lines, respectively. The parent states $\left(3 d_{j_{1}}^{-1} 3 d_{j_{2}}^{-1}\right)_{J}$ [or $\left(3 d_{j}^{-2}\right)_{J}$ in case of $j_{1}=j_{2}$ ] of the $3 d^{-2} n l$ final states are indicated by $\left(j_{1}, j_{2}\right)_{J}$ and the corresponding energy region with $n l=5 d, 6 p, 6 d, 7 p$, and $7 d$ in the order of increasing binding-energy is given by the black or blue horizontal lines in (a). For each parent state the energy positions of the $3 d^{-2} n p$ and $3 d^{-2} n d$ final states are indicated by solid and dashed vertical lines, respectively, and labeled in detail for the parent state $\left(3 d_{3 / 2}^{-2}\right)_{0}$. For the parent states $\left(3 d_{3 / 2}^{-1} 3 d_{5 / 2}^{-1}\right)_{2},\left(3 d_{3 / 2}^{-1} 3 d_{5 / 2}^{-1}\right)_{3},\left(3 d_{5 / 2}^{-2}\right)_{0}$, and $\left(3 d_{3 / 2}^{-2}\right)_{2}$ only part of the final states $3 d^{-2} n l$ are visible and indicated by vertical lines. The vertical arrows in (e,f) indicate the $L_{3} M_{4,5} N_{4,5}$ normal Auger transitions overlapping with the $L_{1} M_{4,5} M_{4,5}$ resonant Auger lines. For both spectra of each threshold the arbitrary units for the Auger intensities are identical.

at $5451.37 \mathrm{eV}$, i.e., on the top of the $2 s \rightarrow 6 p$ resonance, the intensity ratio of $\left(3 d_{3 / 2}^{-1} 3 d_{5 / 2}^{-1}\right)_{4} 6 p$ to $\left(3 d_{3 / 2}^{-1} 3 d_{5 / 2}^{-1}\right)_{4} 7 p$ amounts approximately to $4: 1$ while the ratio at $5452.77 \mathrm{eV}$, i.e., on the top of the $2 s \rightarrow 7 p$ resonance, amounts approximately to $3: 2$. The fact that the Auger transition to the final state $\left(3 d_{3 / 2}^{-1} 3 d_{5 / 2}^{-1}\right)_{4} 6 p$ is the most intense after both the $2 s \rightarrow 6 p$ excitation and the $2 s \rightarrow 7 p$ excitation can be understood readily on the basis of the $2 s$ photoabsorption cross section. 
Actually, the $2 s^{-1} 6 p$ and $2 s^{-1} 7 p$ [13] resonances are split by less than $2 \mathrm{eV}$ while the lifetime broadening amounts to $3.35 \mathrm{eV}$ [29] so that the resonances overlap significantly. As a result, the $2 s^{-1} 7 p$ resonance has already significant intensity at the maximum of the $2 s^{-1} 6 p$ resonance. Because of the fact that the $2 s^{-1} 6 p$ resonance is much more intense than the $2 s^{-1} 7 p$ resonance, its cross section is still dominating at the position of the maximum of the latter resonance; Brown et al. [31] have shown a similar behavior for the resonances $2 p_{3 / 2}{ }^{-1} 5 d$ and $2 p_{3 / 2}{ }^{-1} 6 d$ by deconvoluting the $L_{3}$ photoabsorption cross sections. These observations readily explain the dominance of the Auger transition to the final state $\left(3 d_{3 / 2}^{-1} 3 d_{5 / 2}^{-1}\right)_{4} 6 p$ as well as the relative increase of the transition to the final state $\left(3 d_{3 / 2}^{-1} 3 d_{5 / 2}^{-1}\right)_{4} 7 p$ at the energy position of the $2 s^{-1} 7 p$ resonance. In addition to the given cross section argument in the photoabsorption cross section, the intensities of the Auger transitions are also influenced by shake-up or shake-down effects as well as electronic-lifetime-interference contributions; see Ref. [13].

In the experimental spectrum of Fig. 8(f) an additional shoulder at a binding energy of approximately $1419 \mathrm{eV}$ is visible and not reproduced by theory. Based on the $Z+2$ approximation using the term values of $\mathrm{Ba}$ II, this energy position is too high for an assignment to the final state $\left(3 d_{3 / 2}^{-1} 3 d_{5 / 2}^{-1}\right)_{4} 8 p$, in particular since the shoulder extends up to the binding energy of the $\left(3 d_{3 / 2}^{-1} 3 d_{5 / 2}^{-1}\right)_{4}$ parent state so that we assume that this shoulder is formed by different $\left(3 d_{3 / 2}^{-1} 3 d_{5 / 2}^{-1}\right)_{4} n p$ final states with $n \geqslant 8$.

In addition to the two dominating resonant Auger transitions discussed up to now, Figs. 8(e) and 8(f) also display resonant Auger transitions related to other $3 d^{-2}$ parent states, however, with significantly less intensity. Nevertheless, for all these parent states the intensity ratios of the Auger decays to the final states $3 d^{-2} 6 p$ and $3 d^{-2} 7 p$ show a similar behavior.

The resonant Auger transitions of the $2 p_{1 / 2} \rightarrow 5 d$ excitations at $5105.4 \mathrm{eV}$ photon energy and $2 p_{1 / 2} \rightarrow 6 d$ transition at $5106.6 \mathrm{eV}$ photon energy are shown in Figs. 8(c) and 8(d), respectively. The strongest spectral features are the Auger transitions to the final states $\left(3 d_{3 / 2}^{-1} 3 d_{5 / 2}^{-1}\right)_{4} 5 d,\left(3 d_{3 / 2}^{-1} 3 d_{5 / 2}^{-1}\right)_{4} 6 d$, and $\left(3 d_{3 / 2}^{-1} 3 d_{5 / 2}^{-1}\right)_{4} 7 d$ visible in the binding-energy region from 1410 to $1420 \mathrm{eV}$. In Fig. $8(\mathrm{c})$ the $2 p_{1 / 2}^{-1} \rightarrow\left(3 d_{3 / 2}^{-1} 3 d_{5 / 2}^{-1}\right)_{4} 5 d$ transition is approximately twice as intense as the $2 p_{1 / 2}^{-1} \rightarrow$ $\left(3 d_{3 / 2}^{-1} 3 d_{5 / 2}^{-1}\right)_{4} 6 d$ transition, while the latter one seems to be as intense as the $2 p_{1 / 2}^{-1} \rightarrow\left(3 d_{3 / 2}^{-1} 3 d_{5 / 2}^{-1}\right)_{4} 7 d$ transition. Contrary to this, in Fig. $8(\mathrm{~d})$ the $2 p_{1 / 2}^{-1} \rightarrow\left(3 d_{3 / 2}^{-1} 3 d_{5 / 2}^{-1}\right)_{4} 5 d$ transition is only approximately 1.3 times as intense as the $2 p_{1 / 2} \rightarrow\left(3 d_{3 / 2}^{-1} 3 d_{5 / 2}^{-1}\right)_{4} 6 d$ transition, while the $2 p_{1 / 2}^{-1} \rightarrow$ $\left(3 d_{3 / 2}^{-1} 3 d_{5 / 2}^{-1}\right)_{4} 6 d$ transition now seems to be more intense than the $2 p_{1 / 2}^{-1} \rightarrow\left(3 d_{3 / 2}^{-1} 3 d_{5 / 2}^{-1}\right)_{4} 7 d$ transition. The reducing $2 p_{1 / 2}^{-1} \rightarrow\left(3 d_{3 / 2}^{-1} 3 d_{5 / 2}^{-1}\right)_{4} 6 d$ to $2 p_{1 / 2}^{-1} \rightarrow\left(3 d_{3 / 2}^{-1} 3 d_{5 / 2}^{-1}\right)_{4} 7 d$ intensity ratio as a function of increasing photon energy is contrary to the expectation that the ratio increases with the photon energy. However, the observation can readily be understood by taking into account that the $2 p_{1 / 2}^{-1} \rightarrow\left(3 d_{3 / 2}^{-1} 3 d_{5 / 2}^{-1}\right)_{4} 7 d$ Auger transition overlaps almost perfectly with the $2 p_{1 / 2}^{-1} \rightarrow$ $\left(3 d_{3 / 2}^{-2}\right)_{2} 5 d$ Auger transition. Obviously, the decrease of the intensity of the $2 p_{1 / 2}^{-1} \rightarrow\left(3 d_{3 / 2}^{-2}\right)_{2} 5 d$ Auger transition from the spectrum in Figs. 8(c) and $8(d)$ is larger than the expected increase of the $2 p_{1 / 2}^{-1} \rightarrow\left(3 d_{3 / 2}^{-1} 3 d_{5 / 2}^{-1}\right)_{4} 7 d$ Auger transition so that the combined intensity decreases, leading to the abovedescribed observation.

As in the case of Fig. 8(f), at a binding energy of $1419 \mathrm{eV}$, there is a shoulder in the experimental data of Fig. 8(d) that is not reproduced by theory. In analogy to Fig. 8(f) we assign these data to the $\left(3 d_{3 / 2}^{-1} 3 d_{5 / 2}^{-1}\right)_{4}$ nd final states with $n \geqslant 8$. In addition to the dominating transitions to the $\left(3 d_{3 / 2}^{-1} 3 d_{5 / 2}^{-1}\right)_{4}$ nd final states, the transitions to the $\left(3 d_{3 / 2}^{-1} 3 d_{5 / 2}^{-1}\right)_{2}$ nd states are clearly visible. The Auger transitions to final states with other parent states are only of minor importance.

Finally, in Figs. 8(a) and 8(b) the resonant Auger spectra subsequent to the $2 p_{3 / 2} \rightarrow 5 d$ excitations at $4784.9 \mathrm{eV}$ photon energy and $2 p_{3 / 2} \rightarrow 6 d$ transition at $4785.9 \mathrm{eV}$ photon energy are displayed. These spectra are the most complex ones visible in Fig. 8 since in the Auger decay after $2 p_{3 / 2}^{-1}$ ionization almost all $3 d^{-2}$ parent states are significantly populated (see Fig. 6 for comparison). This results in particular at binding energies of 1396,1402 , and $1407 \mathrm{eV}$ in spectral features that consist of a number of different transitions as indicated by the dashed vertical lines. In Figs. 8(a) and 8(b) the intensity ratio for the Auger transitions to the $\left(3 d_{3 / 2}^{-1} 3 d_{5 / 2}^{-1}\right)_{4} 6 d$ and the $\left(3 d_{3 / 2}^{-1} 3 d_{5 / 2}^{-1}\right)_{4} 7 d$ final states seems to be almost unchanged although the primary excitations are different. As already discussed above for the $L_{1}$ and $L_{2}$ excitations, this can be explained by the influence of the Auger transition to the $\left(3 d_{3 / 2}^{-1} 3 d_{3 / 2}^{-1}\right)_{2} 5 d$ final state. In Fig. 8(b) we once again observe in the experimental spectrum a shoulder at a binding energy of $1419 \mathrm{eV}$, which is assigned to transitions to the $\left(3 d_{3 / 2}^{-1} 3 d_{5 / 2}^{-1}\right)_{4}$ nd final states with $n \geqslant 8$; see above.

\section{SUMMARY AND CONCLUSIONS}

We have presented in a comprehensive analysis the $L M M$, $L M N$, and $L N N$ Auger spectra of Xe over a wide kineticenergy range, from 2500 to $4700 \mathrm{eV}$, both experimentally and theoretically. These spectra show an excellent agreement when the lifetime of the final states is taken into account. This is necessary since the lifetime broadenings of the two-hole final states vary significantly so that they are in many cases not negligible compared to the broadenings of the $L$ holes of $\cong 3 \mathrm{eV}$. As a general discussion, we can underline the fact that the Auger spectra are extremely rich in features consisting of about 400 diagram lines, due to the proximity of the three ionized core levels. The calculations clearly demonstrate that a vast majority of the two-hole final states are described in the most adequate way by using $j j$ coupling. The most evident result is that to correctly interpret the Auger spectra it is necessary to take into account all open edges at a given photon energy, as is clearly shown in all (c) panels from Figs. 3-7. The latter panels are related to the relaxation of the $L_{1}$ edge, but contributions from lower-binding-energy thresholds ( $L_{2}$ and $L_{3}$ ) are clearly evidenced by the calculations. This observation is due to similar splitting between the $L$-shell initial-state core holes as well as the $M$-shell and $N$-shell final-state core holes of typically several hundred eV. We are also confident in stating that our state-of-the-art experimental 
conditions and high-level theoretical calculations allow us to perform a spectral assignment with unprecedented detail for the relaxation of such deep core holes.

In addition, we presented the full range of resonant $L M_{4,5} M_{4,5}$ Auger transitions subsequent to the $2 s \rightarrow 6 p, 7 p$ and $2 p \rightarrow 5 d, 6 d$ excitations. The detailed assignment of the transitions to the $\left(3 d_{j_{1}}^{-1} 3 d_{j_{2}}^{-1}\right)_{J} n l$ final states is in particular for the $L_{3}$ core hole rather complex and it allows understanding some intensity ratios that are surprising in a superficial consideration.

In summary, we systematically studied the diagram lines in the $L$-shell Auger spectra of xenon. These results may serve as a reference for more detailed studies performed for part of the spectra, like, e.g., Auger decays of satellites in the photoelectron spectrum; these transitions were avoided in the present study by choosing proper ionization energies. Moreover, the results let us expect rich $L$-shell Auger spectra of other atoms with an atomic charge $Z$ close to 54 , like iodine or cesium.

\section{ACKNOWLEDGMENTS}

Experiments were performed on the GALAXIES beamline at SOLEIL Synchrotron, France (Proposal No. 99160189). We are grateful to the SOLEIL staff for smoothly running the facility. A.F.L. thanks Coordenação de Aperfeiçoamento de Pessoal de Nível Superior-Brazil for the support.
[1] J.-P. Rueff, J. M. Ablett, D. Céolin, D. Prieur, T. Moreno, V. Balédent, B. Lassalle-Kaiser, J. E. Rault, M. Simon, and A. Shukla, J. Synchrotron Radiat. 22, 175 (2015).

[2] D. Céolin, J. M. Ablett, D. Prieur, T. Moreno, J.-P. Rueff, T. Marchenko, L. Journel, R. Guillemin, B. Pilette, T. Marin, and M. Simon, J. Electron Spectrosc. Relat. Phenom. 190, 188 (2013).

[3] A. Kivimäki, A. Naves de Brito, S. Aksela, H. Aksela, O.-P. Sairanen, A. Ausmees, S. J. Osborne, L. B. Dantas, and S. Svensson, Phys. Rev. Lett. 71, 4307 (1993).

[4] M. Simon, R. Püttner, T. Marchenko, R. Guillemin, R. K. Kushawaha, L. Journel, G. Goldsztejn, M. N. Piancastelli, J. M. Ablett, J.-P. Rueff, and D. Céolin, Nat. Commun. 5, 4069 (2014).

[5] R. Püttner, G. Goldsztejn, D. Céolin, J.-P. Rueff, T. Moreno, R. K. Kushawaha, T. Marchenko, R. Guillemin, L. Journel, D. W. Lindle, M. N. Piancastelli, and M. Simon, Phys. Rev. Lett. 114, 093001 (2015).

[6] G. Goldsztejn, T. Marchenko, R. Püttner, L. Journel, R. Guillemin, S. Carniato, P. Selles, O. Travnikova, D. Céolin, A. F. Lago, R. Feifel, P. Lablanquie, M. N. Piancastelli, F. Penent, and M. Simon, Phys. Rev. Lett. 117, 133001 (2016).

[7] S. Carniato, P. Selles, P. Lablanquie, J. Palaudoux, L. Andric, M. Nakano, Y. Hikosaka, K. Ito, T. Marchenko, O. Travnikova, G. Goldsztejn, L. Journel, R. Guillemin, D. Céolin, M. Simon, M. N. Piancastelli, and F. Penent, Phys. Rev. A 94, 013416 (2016).

[8] M. N. Piancastelli, G. Goldsztejn, T. Marchenko, R. Guillemin, R. K. Kushawaha, L. Journel, S. Carniato, J.-P. Rueff, D. Céolin, and M. Simon, J. Phys. B 47, 124031 (2014).

[9] O. Travnikova, T. Marchenko, G. Goldsztejn, K. Jänkälä, N. Sisourat, S. Carniato, R. Guillemin, L. Journel, D. Céolin, R. Püttner, H. Iwayama, E. Shigemasa, M. N. Piancastelli, and M. Simon, Phys. Rev. Lett. 116, 213001 (2016).

[10] O. Travnikova, N. Sisourat, T. Marchenko, G. Goldsztejn, R. Guillemin, L. Journel, D. Céolin, I. Ismail, A. F. Lago, R. Püttner, M. N. Piancastelli, and M. Simon, Phys. Rev. Lett. 118, 213001 (2017).

[11] R. Guillemin, S. Sheinerman, R. Püttner, T. Marchenko, G. Goldsztejn, L. Journel, R. K. Kushawaha, D. Céolin, M. N. Piancastelli, and M. Simon, Phys. Rev. A 92, 012503 (2015).
[12] D. Céolin, T. Marchenko, R. Guillemin, L. Journel, R. K. Kushawaha, S. Carniato, S.-M Huttula, J.-P. Rueff, G. B. Armen, M. N. Piancastelli, and M. Simon, Phys. Rev. A 91, 022502 (2015).

[13] R. K. Kushawaha, K. Jänkälä, T. Marchenko, G. Goldsztejn, R. Guillemin, L. Journel, D. Céolin, J.-P. Rueff, A. F. Lago, R. Püttner, M. N. Piancastelli, and M. Simon, Phys. Rev. A 92, 013427 (2015).

[14] G. Goldsztejn, T. Marchenko, D. Céolin, L. Journel, R. Guillemin, J.-P. Rueff, R. K. Kushawaha, R. Püttner, M. N. Piancastelli, and M. Simon, Phys. Chem. Chem. Phys. 18, 15133 (2016).

[15] G. Goldsztejn, R. Püttner, L. Journel, R. Guillemin, O. Travnikova, R. K. Kushawaha, B. Cunha de Miranda, I. Ismail, D. Céolin, M. N. Piancastelli, M. Simon, and T. Marchenko, Phys. Rev. A 95, 012509 (2017).

[16] M. N. Piancastelli, K. Jänkäla, L. Journel, T. Gejo, Y. Kohmura, M. Huttula, M. Simon, and M. Oura, Phys. Rev. A 95, 061402(R) (2017),

[17] R. Püttner, D. Céolin, R. Guillemin, R. K. Kushawaha, T. Marchenko, L. Journel, M. N. Piancastelli, and M. Simon, Phys. Rev. A 93, 042501 (2016).

[18] L. Avaldi, G. Dawber, R Camilloni, G. C. King, M. Rope, M. R. F. Siggels, G. Stefani, and M. Zitnik, J. Phys. B 27, 3953 (1994).

[19] M. Breinig, M. H. Chen, G. E. Ice, F. Parente, B. Crasemann, and G. S. Brown, Phys. Rev. A 22, 520 (1980).

[20] NIST Atomic Spectra Database, https://www.nist.gov/pml/ atomic-spectra-database.

[21] P. Jönsson, X. He, C. Froese Fischer, and I. P. Grant, Comput. Phys. Commun. 177, 597 (2007).

[22] F. A. Parpia, C. Froese Fischer, and I. P. Grant, Comput. Phys. Commun. 94, 249 (1996).

[23] S. Fritzsche, C. F. Fischer, and G. Gaigalas, Comput. Phys. Commun. 148, 103 (2002).

[24] J. Niskanen, K. Jänkälä, M. Huttula, and A. Föhlisch, J. Chem. Phys. 146, 144312 (2017).

[25] S. Fritzsche, Comput. Phys. Commun. 183, 1525 (2012).

[26] E. G. Berezhko and N. M. Kabachnik, J. Phys. B: At., Mol. Phys. 10, 2467 (1977). 
[27] See Supplemental Material at http://link.aps.org/supplemental/ 10.1103/PhysRevA.96.022501 for detailed information on the theoretical results of the Auger transitions including assignments, energy positions, and intensities.

[28] X-ray Data Booklet, Center for X-ray Optics and Advanced Light Source, Lawrence Berkeley National Laboratory, 2009, http://xdb.lbl.gov/.

[29] M. H. Chen, B. Crasemann, and H. Mark, Phys. Rev. A 24, 177 (1981).

[30] L. Asplund, P. Kelfve, B. Blomster, H. Siegbahn, and K. Siegbahn, Phys. Scr. 16, 268 (1977).
[31] G. S. Brown, M. H. Chen, B. Crasemann, and G. E. Ice, Phys. Rev. Lett. 45, 1937 (1980).

[32] G. B. Armen, S. H. Southworth, J. C. Levin, U. Arp, T. LeBrun, and M. A. MacDonald, Phys. Rev. A 56, R1079 (1997).

[33] A. Messiah, Quantum Mechanics (Elsevier, Amsterdam, 1961), Vol. II, pp. 1065.

[34] G. B. Armen, J. Tulkki, T. Åberg, and B. Crasemann, Phys. Rev. A 36, 5606 (1987).

[35] This value differs from that given in Ref. [13] and is due to the correction of a slight calibration error in the latter reference. 